\title{
Numerical Simulations of Electrically Induced Birefringence in Photonic Liquid Crystal Fibers
}

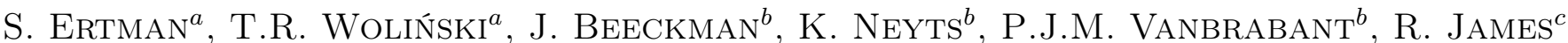
AND F.A. FERNÁNDEZ ${ }^{c}$

${ }^{a}$ Faculty of Physics, Warsaw University of Technology, Koszykowa 75, 00-662 Warsaw, Poland

${ }^{b}$ Liquid Crystals and Photonics Group, Ghent University, Ghent, Belgium

${ }^{c}$ Department of Electronic \& Electrical Engineering, University College London, United Kingdom

It has been recently experimentally demonstrated that propagation and polarization properties of the photonic liquid crystal fibers can be effectively tuned with the electric field. In particular, effective electric tuning of the phase birefringence has been obtained in the photonic liquid crystal fibers based on the high index glasses. Accurate numerical simulations of the impact of electric field on the guiding properties of the photonic liquid crystal fibers require complex methods, in which all important physical properties of the liquid crystal are taken into account (optical anisotropy, molecular orientation and relatively high losses). In this paper we present two different numerical approaches based on the finite element method. First one utilizes the simplified assumption of the "collective tilt" of molecules, and gives rough estimation of the phase birefringence tuning range. The second approach is much more rigorous, since electrically induced reorientation of the liquid crystal is calculated with a Q-tensor method giving the values of the inhomogeneous permittivity tensor. The value of the electrically induced birefringence calculated with the second method is in a good agreement with the experimental results.

PACS numbers: 42.81.Gs, 78.20.Bh, 42.79.Kr

\section{Introduction}

In the last few years a lot of research activities all over the world have been focused on photonic crystal fibers (PCFs) with an array of micro-holes running along the axial direction. By changing both: the position and diameters of the micro-holes it is possible to tailor the optical properties in such fibers, allowing for example for useful nonlinear properties, thermally independent birefringence or so-called endless single-mode propagation [1].

It is also possible to tune properties of the PCF by filling their micro-holes with various substances [2]. Liquid crystals (LCs) are a very good candidate for making tunable in-fiber devices since they exhibit very high electro-optic and thermo-optic effects. For example, it was possible to demonstrate thermal tuning of the photonic band-gap position [3, 4], electric tuning of the transmission spectra in hollow-core [5] and in silica glass-core photonic liquid crystal fibers (PLCFs) [6]. Thermal tuning of the PLCF guiding mechanism was presented by using a special LC mixture with the ordinary refractive index lower (in a certain temperature range) than the refractive index of the silica glass $[7,8]$. Also tunable birefringence has been recently demonstrated in the band-gap guiding PLCFs $[9,10]$ as well as in the low-loss index-guiding PLCFs based on the high index glass PCFs [11].

There was also a number of theoretical works discussing propagation and polarization properties of PCFs filled with LCs, confirming that their properties strongly rely on $\mathrm{LC}$ refractive indices and molecular orientation
[12-15]. Also, liquid crystal attenuation can play a significant role, especially if a guided mode is penetrating LC-filled holes [16]. Probably the most advanced numerical analysis was presented in [17, 18], where the alignment of the biased liquid crystal was simulated for different values of electric field, and then transmission spectra of the PLCF were calculated.

In this paper we use similar two-step approach to model phase birefringence tuning in the PLCF. At the first step reorientation of the molecules caused by the transverse electric field is calculated by using algorithms described in [18], and then guided modes are found with a full-vector mode solver presented in [19]. The results obtained show a good agreement with the experimental data. Additionally, we present results obtained with a simplified model, which can be used in the case if only rough estimation of the tuning range of phase birefringence is needed.

\section{Experimental background and motivation}

In our investigations we have decided to model a real PLCF structure described in detail in [11]. Here we will give only main information about the experiment. The host PCF is made of the PBG08 glass with a very high refractive index $(\approx 1.95)$ and it is filled with the $5 \mathrm{CB}$ nematic liquid crystal. Refractive indices of the PBG08 glass and 5CB are shown in Fig. 1a. Since the refractive index of the glass is higher than both ordinary and extraordinary indices of the LC, it is obvious that index-guiding propagation was observed with losses 
of $0.19 \mathrm{~dB} / \mathrm{cm}$ (much lower than in band-gap guiding PLCFs reported so far). The fiber diameter is $125.4 \mu \mathrm{m}$ and it consists of six rings of the holes with diameters of $5.2 \mu \mathrm{m}$ and is characterized by the pitch (distance between holes) equal to $7.6 \mu \mathrm{m}$ (Fig. 1b).

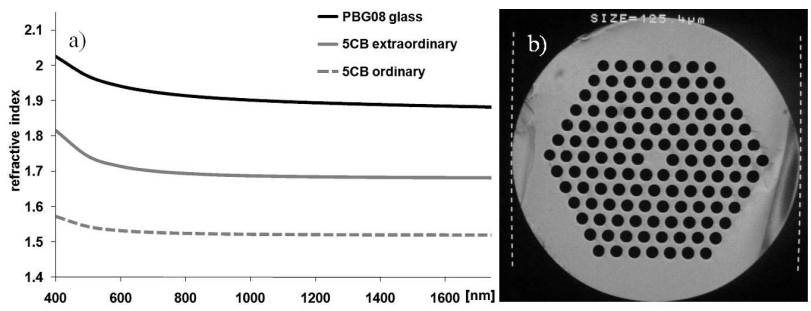

Fig. 1. Dispersion characteristics of the PBG08 glass and 5CB liquid crystal (a) and cross-section of the PCF14(6) microstructured fiber (b).

In our experiment the PLCF was placed between two flat electrodes and it has been noticed that changes in the electric field intensity led to continuous and repeatable changes in the state of polarization (SOP) at the output. It means that continuous changes of the phase birefringence have been electrically induced by LC molecules reorientation (Fig. 2). Phase birefringence tuning range for

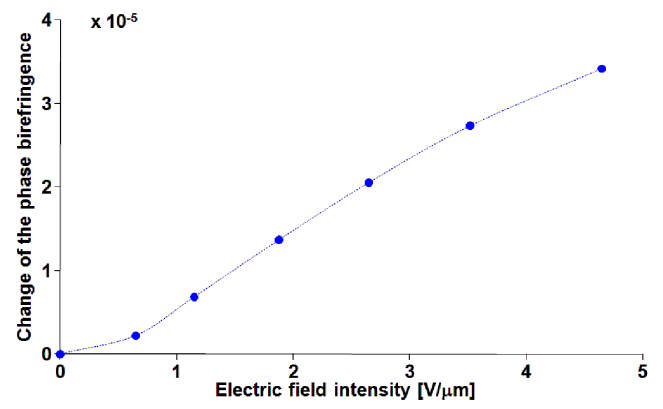

Fig. 2. Phase birefringence tuning in the PCF14(6) filled with 5CB (measured for wavelength $1640 \mathrm{~nm}$ ).

PCF filled with $5 \mathrm{CB}\left(\approx 4 \times 10^{-5}\right)$ was lower than for samples filled with LCs with higher optical anisotropy [10]. In this paper we decided to model theoretically the PLCF based on the $5 \mathrm{CB}$ LC, since all the physical properties of this material are well described in literature, which means that accurate calculations of LC alignment are possible which allows for direct validation of the results.

\section{Collective tilt model}

In this section we will present a simplified model. In this model collective tilt of liquid crystal molecules is assumed, which means that all molecules have the same tilt angle. If the tilt angle is equal to $0^{\circ}$ (planar alignment - Fig. 3a), birefringence of the PLCF is also equal to 0 . In contrast, if all molecules are aligned perpendicularly to the fiber axis (Fig. 3b), the phase birefringence of the $\mathrm{PLCF}$ reaches maximum.

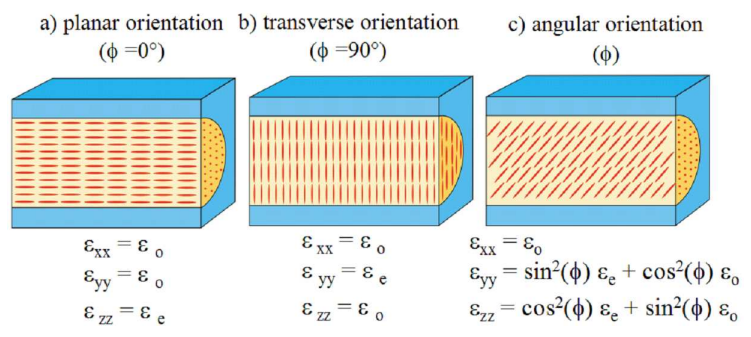

Fig. 3. LC molecules orientations discussed in a theoretical model and corresponding components of the diagonal dielectric permittivity tensor: (a) planar, (b) transverse, (c) tilted.

For both planar and transverse alignment dielectric permittivity is expressed by the diagonal tensor $\varepsilon=\left[\varepsilon_{x x}, \varepsilon_{y y}, \varepsilon_{z z}\right]$. In fact, also for tilted alignment it is possible to use diagonal tensor (as expressed at Fig. 3c), because mixed components $\varepsilon_{y z}=\varepsilon_{z y}=$ $\left(\varepsilon_{\mathrm{e}}-\varepsilon_{\mathrm{o}}\right) \sin (\phi) \cos (\phi)$ are relatively small, when compared to the diagonal components. Actually our calculations performed with full and diagonal tensor showed that neglecting mixed components causes that calculated effective indices of the guided modes differ only by about $1 \times 10^{-6}$

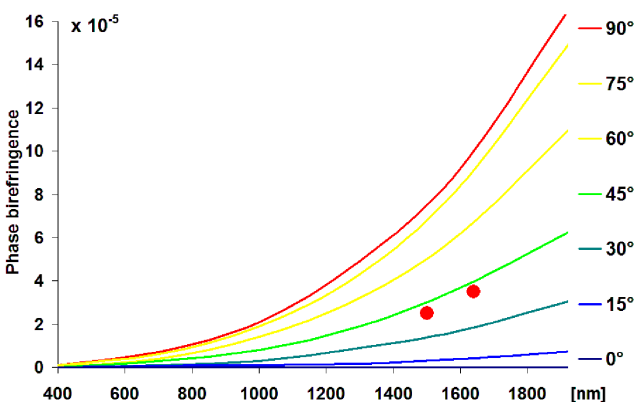

Fig. 4. Numerical simulations of the phase birefringence tuning in the PCF14(6) filled with 5CB induced by collective tilt of the LC molecules. The red dots indicate maximal tuning range obtained in experiment for two different wavelengths [11].

It is obvious that collective reorientation of the LC molecules has never been observed in real situations, as well as uniform transverse reorientation of all molecules. However, such approach allows for quick quantitative estimation of the maximal tuning range. For example for the PCF14(6) filled with 5CB theoretically it is possible to tune phase birefringence from 0 to $1 \times 10^{-4}$, since in our experiments we were able to obtain tuning range limited to $4 \times 10^{-5}$ (Fig. 4). The difference is noticeable, but collective tilt model could be applied for time effective modeling of PLCF structures. Usually there is a quite big number of parameters that should be scanned (i.e. refractive indices of glass and liquid crystal, holes diameter and spacing, operating wavelength ...), so in the first approximation it is reasonable to use this simple and fast model. When some optimal structures are 
found, there will be a space for more rigorous methods, as described in the next section.

\section{Rigorous modeling of electrically induced birefringence}

To perform accurate modeling of the electrically induced birefringence in the PLCFs the calculations should be divided into two steps: first alignment of the LC should be found and afterwards a mode-solver should be applied. In our investigations variable order calculations were based on the minimization of the Landaude Gennes free energy functional [18]. Then full-vector finite-element mode solver was used, and it was able to incorporate the full anisotropy of the materials [18]. It is worth to mention that both calculation steps was directly coupled and worked on the same finite-element meshes.

Figure 5 shows two kind of geometries that were used in our work. First one consists of the whole structure of the PLCF placed between two flat electrodes, and as it could be seen a mesh is graded in such a way that there is an increased number of the elements in the central part of the fiber. It is a typical situation for the PCF calculations, since the mode field is strongly localized in the core region. However for the LC alignment calculations such a mesh is not optimal, because for outer rings of holes the mesh is quite coarse. If the outer rings mesh is too coarse, there is a risk that calculated alignment of LC will be different in the central and outer holes, even if the electric field intensity in all holes would be comparable (actually, such a situation was observed in our calculations). This issue could be solved by using the mesh with extended number of the elements, to ensure uniform density of elements in every hole. However such solution have two drawbacks - it increases computation time, and also it is inefficient for the mode-solver.

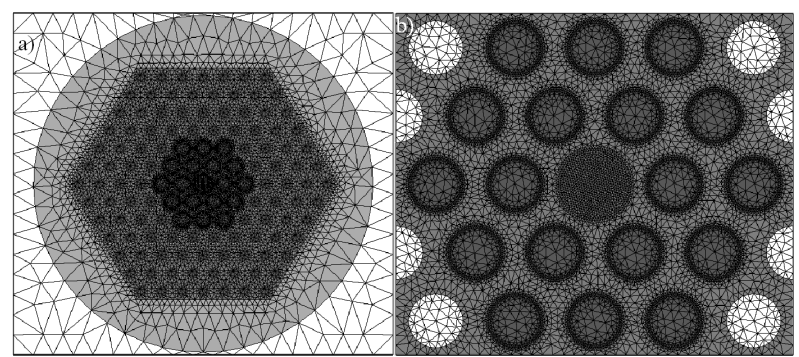

Fig. 5. Two geometries used in our calculations: (a) whole PLCF between electrodes; (b) geometry limited only to central part of the fiber.

An alternative solution is to limit the geometry to only few rings of the holes, as presented in Fig. 5b. In this geometry upper and lower borders represent some "virtual" electrodes. In our case limitation of the number of the holes does not have significant impact on the mode calculations, because the mode is highly localized in the core [11]. Also confinement losses of the fiber are very low and could be neglected [11], so there is no need to apply any absorbing boundary conditions. However it is not clear how "virtual electrodes" will change distribution of the electric field.
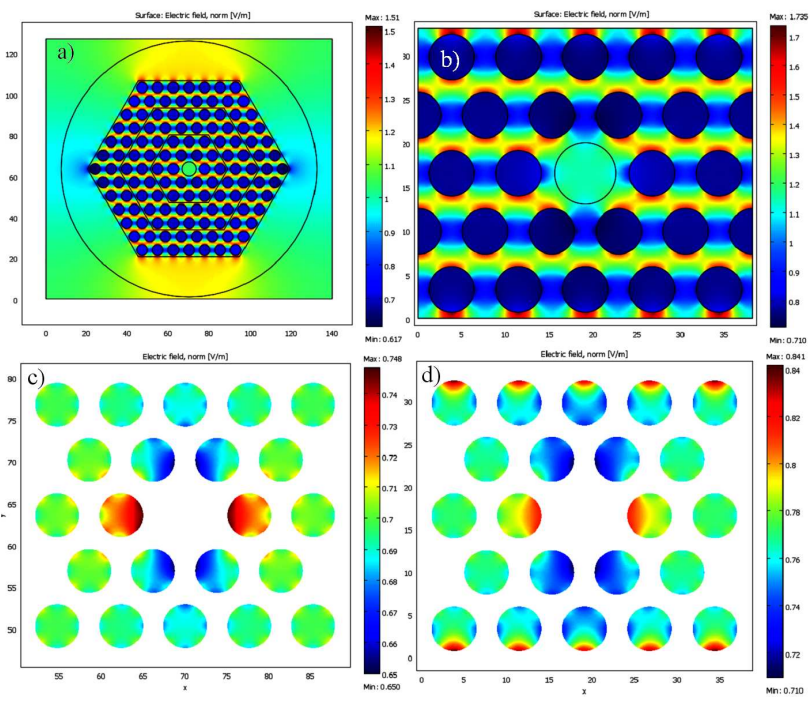

Fig. 6. Electric field distribution for two discussed geometries: whole PLCF between electrodes ( $\mathrm{a}$ and c) and limited geometry (b and d).

To check if it is possible to use limited geometry for director calculations without the risk that the electric field will be deformed, we have made some additional calculations. Figure 6 presents normalized electric field intensity calculated for both geometries. Figures $6 \mathrm{a}$ and $\mathrm{b}$ shows whole structures, since Fig. 6c and d show intensity only in two central rings of holes. It could be noticed that electric field distributions in both cases are quite similar (especially in the most inner ring of holes, which mostly determines properties of the PLCF modes).

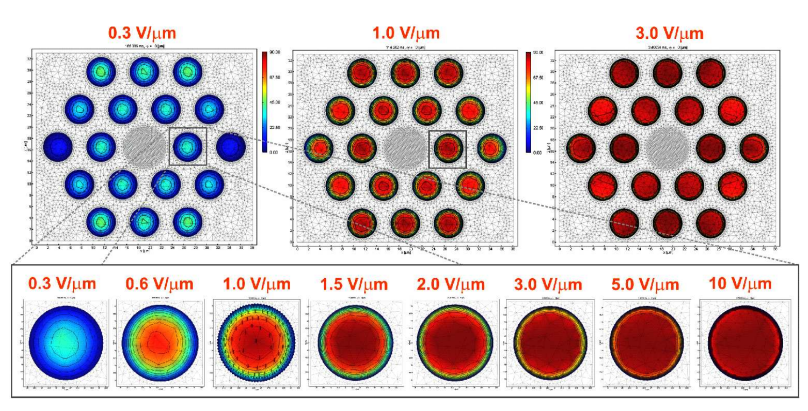

Fig. 7. Alignment of LC molecules for different intensities of electric field.

Examples of the calculated alignment of LC molecules are presented in Fig. 7 and Fig. 8. In both cases a color map represents a tilt angle of the molecules. It should be mentioned that in our calculations we were using fixed boundary conditions on the holes borders, which means that the molecules on the walls are always parallel to the 
fiber axis.

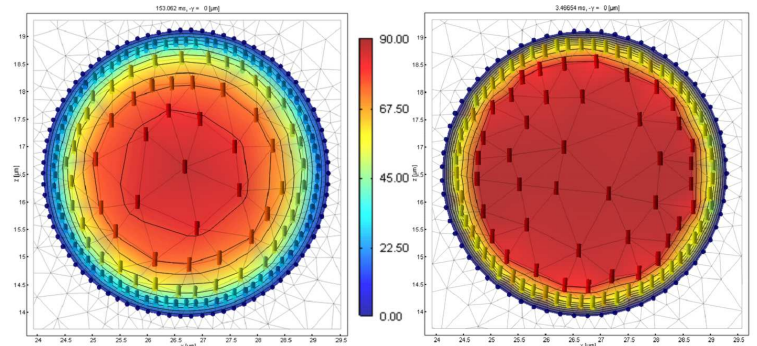

Fig. 8. Alignment of LC molecules in the one selected hole for two different intensities of electric field (left side: $1 \mathrm{~V} / \mu \mathrm{m}$, right side: $3 \mathrm{~V} / \mu \mathrm{m})$.

Calculations of LC alignment was performed for different values of the electric field intensity, and each time mode-solver was applied to find birefringence of the PLCF (Fig. 9). First series of calculation was done for the mesh with 10 thousands (10k) of elements and a good agreement with experimental results was observed for low intensities, but for higher intensities the calculated birefringence was smaller than this observed in a real fiber. This could be explained by the fact that fixed boundary conditions were defined at the edges of holes (strong anchoring) and as a result reorientation of the molecules in nodes close to the boundaries was somehow limited. This effect could be minimized by defining a finer mesh close to the hole edges, since it decreases the area with "limited" reorientation. The results obtained for the mesh of $17 \mathrm{k}$ elements were consistent with the experiment in the wider range than for 10k elements (Fig. 9). It could be expected that a further increase of the mesh density would lead to additional extension of the range of agreement. Unfortunately it is computationally expansive, because calculation time would be much longer (single calculation of modes at the specified electric field intensity for $10 \mathrm{k}$ mesh lasted for about $16 \mathrm{~min}$, for $13 \mathrm{k}$ it was about $33 \mathrm{~min}$ and for $17 \mathrm{k}$ about 85 min was needed).

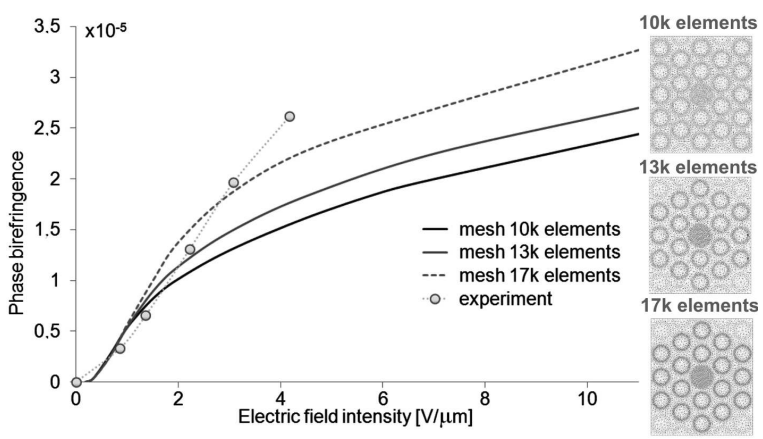

Fig. 9. Phase birefringence tuning calculated with three meshes with different densities of elements close to the hole edges.

As an alternative method to expansive extension of the mesh we are planning to use numerical model in which reorientation of molecules at the boundaries will be also possible (weak anchoring). We expect that it will be possible to have agreement with experiment in much wider range of the electric field intensities.

\section{Conclusions}

We have presented calculations of the phase birefringence tuning in the PLCF with two numerical models. Simplified model with assumption of the collective tilt can be used for fast and rough modeling of desirable tuning range. Rigorous model with the alignment determination can be applied for detailed analysis, giving good agreement with real structures.

\section{Acknowledgments}

This work was supported by the Polish Ministry of Science and Education under the grant N517 056535 and partially by the "Master" Programme of the Polish Science Foundation. Part of this research was carried out in the framework of the project on light scattering at Ghent University of the Research Foundation - Flanders (FWO).

\section{References}

[1] P.St.J. Russell, Lightwave Technol. 24, 4729 (2006).

[2] C. Kerbage, R.S. Windeler, B.J. Eggleton, P. Mach, M. Dolinski, J.A. Rogers, Opt. Commun. 204, 179 (2002).

[3] T. Larsen, A. Bjarklev, D. Hermann, J. Broeng, Opt. Express 11, 2589 (2003).

[4] T.R. Wolinski, K. Szaniawska, K. Bondarczuk, P. Lesiak, A.W. Domanski, R. Dabrowski, E. Nowinowski-Kruszelnicki, J. Wojcik, Opto-Electronics Rev. 13, 59 (2005).

[5] Q. Lu, S.T. Wu, Appl. Phys. Lett. 85, 2181 (2005).

[6] M.W. Haakestad, T.T. Alkeskjold, M.D. Nielsen, L. Scolari, J. Riishede, H.E. Engan, A. Bjarklev, IEEE Photon. Technol. Lett. 17, 819 (2005).

[7] T.R. Wolinski, K. Szaniawska, S. Ertman, P. Lesiak, A.W. Domanski, R. Dabrowski, E. Nowinowski-Kruszelnicki, J. Wojcik, Meas. Sci. Technol. 17, 985 (2006).

[8] T. Pustelny, M. Grabka, Acta Phys. Pol. A 114, A-113 (2008).

[9] L. Scolari, T. Alkeskjold, J. Riishede, A. Bjarklev, D. Hermann, A. Anawati, M. Nielsen, P. Bassi, Opt. Express 13, 7483 (2005).

[10] L. Wei, L. Eskildsen, J. Weirich, L. Scolari, T.T. Alkeskjold, A. Bjarklev, Appl. Opt. 48, 497 (2009).

[11] S. Ertman, T.R. Wolinski, D. Pysz, R. Buczynski, E. Nowinowski-Kruszelnicki, R. Dabrowski, Opt. Express 17, 19298 (2009).

[12] D.C. Zografopoulos, E.E. Kriezis, T.D. Tsiboukis, Opt. Express 14, 914 (2006).

[13] J. Sun, C.C. Chan, Opt. Lett. 32, 1989 (2007). 
[14] G. Tartarini, M. Pansera, T.T. Alkeskjold, A. Bjarklev, P. Bassi, J. Lightwave Technol. 25, 2522 (2007).

[15] G. Ren, P. Shum, J. Hu, X. Yu, Y. Gong, J. Lightwave Technol. 26, 3650 (2008).

[16] S. Ertman, T. Nasilowski, T.R. Wolinski, H. Thienpont, Photon. Lett. Poland 1, 13 (2009).

[17] J. Weirich, J. Laegsgaard, L. Scolari, L. Wei, T.T. Alkeskjold, A. Bjarklev, Opt. Express 17, 4442 (2009).
[18] R. James, E. Willman, F.A. Fernández, S.E. Day, IEEE Trans. Electron Dev. 53, 1575 (2006).

[19] J. Beeckman, R. James, F.A. Fernández, W. De Cort, P.J.M. Vanbrabant, K. Neyts, J. Lightwave Technol. 27, 3812 (2009). 